III JORNADAS DE FORMACIÓN DEL PROFESORADO DE EDUCACIÓN FÍSICA: ENCUENTROS CAPAS-Ciudad

Tarbes, 13 de febrero del 2019

\title{
Promoción de la
} actividad física para la salud en la escuela: formación teórica Y práctica

\section{Libro de Actas}

Coordinadores:

Alberto Aibar Solana

Raquel Pérez-Ordás

Sergio Estrada Tenorio 



\section{JORNADAS DE FORMACIÓN DEL PROFESORADO DE EDUCACIÓN FÍSICA: ENCUENTROS CAPAS-Ciudad}

Tarbes, 13 de febrero del 2019

\section{Promoción de la} actividad física para la salud en la escuela: formación teórica Y práctica

\section{Libro de Actas}

\section{Coordinadores:}

Alberto Aibar Solana

Raquel Pérez-Ordás

Sergio Estrada Tenorio

\section{CRÉDITOS}

(C) Alberto Aibar Solana, Raquel Pérez-Ordás y Sergio Estrada Tenorio

$1^{\text {a }}$ Edición, Huesca, 2019

Edita: CAPAS- Cité/CAPAS-Ciudad. Universidad de Zaragoza

ISBN: 978-84-16723-61-4

Imprime: Universidad de Zaragoza. Reservados todos los derechos. Cualquier forma de reproducción, distribución, comunicación pública o transformación de esta obra solo puede ser realizada con la autorización de sus titulares, salvo excepción prevista por la ley. Dirigirse a CEDRO (www.cedro.org) si necesita fotocopiar o escanear algún fragmento de esta obra.

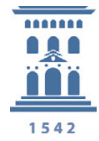

Servicio de Publicaciones

Universidad Zaragoza 


\section{Índice}

PÁGINA

INTRODUCCIÓN: "Promoción de la actividad física para la salud en la escuela: formación teórica y práctica

\section{BLOQUE: ACTIVIDAD FÍSICA EN LA ESCUELA: CONTEXTUALIZACIÓN}

7. Los niveles de actividad física en los niños y los adolescentes en general, en la escuela y en Educación Física.

Julien Bois, Léna Lhuisset, Caroline Bernal y Nicolas Fabre (CAPAS-Cité, Universidad des Pays de l'Adour)

12. La escuela y la promoción de la salud del alumnado.

Carole Sève (Ministerio de Educación Nacional, Francia)

15. La promoción de la actividad física para la salud en Educación Física en España. José Antonio Julian/ Alberto Aibar (Universidad de Zaragoza)

\section{BLOQUE: ACCIONES Y RESULTADOS DE EXPERIENCIAS}

20. Curso teórico puesto en práctica en el Collège de Lourre Barrousse. Ludovic Dolce y Magalie Annecy (Collège Loures Barousse, EPS)

24. Ejemplo de innovación educativa. El trabajo colaborativo: efecto de un sistema de aprendizaje cooperativo para el bienestar psicosocial de los alumnos. Pascal Legrain y Guillaume Escalie (STAPS, Universidad de Burdeos)

26. Intervención escolar para promover la actividad física $y$ disminuir el tiempo de sedentarismo en una población de niños de barrios desfavorecidos de 6 a 11 años. Caroline Bernal, Nicolas Fabre, Léna Lhuisset y Julien Bois (CAPAS-Cité, Université de Pau et des Pays de l'Adour)

\section{BLOQUE: TALLERES PRÁCTICOS}

29. .................... "Descansos" sedentarios. Organización de la clase para disminuir el tiempo sedentario. Actividades "activas" en el aula.

Caroline Bernal, Nicolas Fabre (Université de Pau et des Pays de l'Adour)

33. ..................... Ejemplo de configuración del trabajo colaborativo.

Pascal Legrain y Guillaume Escalie (UF STAPS, Université de Bordeaux)

34. .................... Teoría y práctica de la promoción de la actividad física: Encuentros Intercentros. “El Test de Responsabilidad”. Una estrategia previa a los Encuentros Intercentros. Paz Tierz, Gemma Bermejo, Raquel Pérez-Ordás y Eduardo Generelo (CAPAS-Cité)

37. ..................... Teoría y práctica de la promoción de la AF: Encuentros Intercentros. José Antonio Julián, Javier Sevil y Alberto Aibar (Universidad de Zaragoza)

40. ..................... Reflexiones sobre la organización de situaciones de enseñanza de Educación Física con impacto sobre la salud: trabajo intermitente de alta intensidad en deportes de equipo.

Laurent Souriac (Université de Pau et des Pays de l'Adour) 


\title{
"Promoción de la actividad física para la salud en la escuela: formación teórica $y$ práctica".
}

\author{
Introducción
}

La realización de las III Jornadas de formación del profesorado CAPAS-Ciudad en Tarbes cierra una serie de encuentros formativos dirigidos al personal docente que pretende reforzar la red de formación permanente del profesorado existente a nivel transpirenaico. El proyecto CAPAS-Cité (Centro Pirenaico para la Mejora y Promoción de la Actividad Física para la Salud) tiene como objetivo promover la actividad física y los estilos de vida saludables para con ello mejorar la calidad de vida de la población. La celebración de las III Jornadas de formación del profesorado tituladas "Promoción de la actividad física para la salud en la escuela: formación teórica y práctica”, en Tarbes (Francia), en febrero del 2019, pretende dar respuesta a esa búsqueda de la mejora de la calidad de vida de la población mediante un encuentro donde las experiencias prácticas presentadas, dan voz a los aspectos teóricos que se debaten a nivel nacional e internacional. El espíritu transpirenaico de esta acción, está sustentado en la relación entre los agentes promotores de la actividad física salud entre Francia y España, los cuales llevan colaborando y trabajando juntos durante los últimos años.

Los objetivos de las III Jornadas de formación del profesorado fueron fundamentalmente:

- Fomentar un mayor conocimiento sobre el estado actual de la promoción de la actividad física en los entornos escolares.

- Difundir el conocimiento sobre estrategias efectivas de promoción de la actividad física en centros educativos.

- Permitir reflexiones entre diferentes agentes de diferentes países sobre la forma más efectiva de abordar la promoción de la actividad física y la salud.

En dicha formación, participaron profesores de centros educativos, profesores de educación física en formación inicial, así como los diversos actores del sistema educativo que trabajan en la promoción de la salud del alumnado (personal de gestión, escuela de enfermería, etc.).

Se presentaron comunicaciones, experiencias y talleres vinculados con la temática de la jornada que distribuyeron en tres grandes bloques:

1. Actividad física en la escuela: contextualización.

2. Acciones y resultados de experiencias.

3. Talleres prácticos. 
En el bloque de 'Actividad física en la escuela', se presentaron comunicaciones vinculadas con la temática con los siguientes títulos:

1. Los niveles de actividad física en los niños y los adolescentes en general, en la escuela y en Educación Física.

2. La escuela y la promoción de la salud del alumnado.

3. La promoción de la actividad física para la salud en Educación Física en España.

En el bloque de 'Acciones y resultados de experiencias' se presentaron los siguientes trabajos vinculados con experiencias prácticas aplicadas:

1. Curso teórico puesto en práctica en el collège de Lourre Barrousse

2. Ejemplo de innovación educativa. El trabajo colaborativo: efecto de un sistema de aprendizaje cooperativo para el bienestar psicosocial de los alumnos.

3. Intervención escolar para promover la actividad física y disminuir el tiempo de sedentarismo en una población de niños de barrios desfavorecidos de 6 a 11 años.

Para finalizar las jornadas, se llevaron a cabo el bloque de 'Talleres prácticos' con las siguientes temáticas:

1. "Descansos" sedentarios. Organización de la clase para disminuir el tiempo sedentario. Actividades "activas" en el aula.

2. Ejemplo de configuración del trabajo colaborativo.

3. Teoría y práctica de la promoción de la actividad física: Encuentros Intercentros. "El Test de Responsabilidad". Una estrategia previa a los Encuentros Intercentros.

4. Teoría y práctica de la promoción de la AF: Encuentros Intercentros.

5. Reflexiones sobre la organización de situaciones de enseñanza de Educación Física con impacto sobre la salud: trabajo intermitente de alta intensidad en deportes de equipo.

Este documento pretende recopilar, en formato de acta, de manera sintetizada, todos los trabajos y contribuciones académicas presentadas en cada uno de los tres bloques que conformaban las III Jornadas de formación del profesorado. Confiamos en que este documento sirva como apoyo a docentes y profesionales de la promoción de la actividad física que quieran reciclarse, conocer cómo trabajan otros profesionales y, en definitiva, seguir formándose en la promoción de la actividad física para la mejora de la salud.

Coordinadores del documento

Alberto Aibar Solana, Raquel Pérez-Ordás y Sergio Estrada Tenorio 


\section{Los niveles de actividad física en los niños $y$ los adolescentes en general, en la escuela y en Educación Física.}

Julien Bois', Léna Lhuisset', Caroline Bernal' y Nicolas Fabre' 'Université de Pau et des Pays de l'Adour, Laboratoire Mouvement Equilibre Performance Santé (EA 4445).

\section{Introducción}

El propósito de esta comunicación es presentar el estado del conocimiento sobre los niveles de actividad física y sedentarismo de niños y adolescentes. Estos datos se presentan para una jornada completa, posteriormente se detallan para el contexto específico de la escuela y, finalmente, para la clase de Educación Física. En la medida de lo posible, primero se presentan los datos globales, luego los datos específicos de Francia y, finalmente, los datos locales que hemos observado en los estudios realizados en Tarbes y su región, o en el marco de las colaboraciones con compañeros de la Universidad de Zaragoza.

Antes de presentar las cifras, recordaremos primero las definiciones de los conceptos clave utilizados para la jornada global, luego en el contexto escolar y, finalmente, en el contexto específico de la clase de Educación Física.

\section{Definiciones de términos:}

La actividad física (AF) se define como "cualquier movimiento del cuerpo producido por la contracción de los músculos esqueléticos que conlleva un aumento del gasto energético en relación con el gasto energético en reposo" (Caspersen, Powell, \& Christenson, 1985). Es el componente más variable del gasto total de energía de un individuo. La AF reúne todas las actividades que se pueden practicar en diferentes contextos persiguiendo varios objetivos (utilitarios, sanitarios, sociales, etc.). Las principales áreas de práctica de la AF son el trabajo, los transportes, las actividades domésticas y el ocio. Estos últimos incluyen el ejercicio, el deporte y la AF de ocio no estructurada.

El interés de un concepto tan amplio es considerar los cambios de comportamientos que no se limiten a las actividades exclusivas de ocio y organizadas, sino que también puedan estar relacionadas con la organización del tiempo escolar, del trabajo en clase o con los modos de transporte.

El comportamiento sedentario o sedentarismo se refiere a las actividades que no aumentan significativamente el gasto de energía por encima del nivel de descanso e incluye actividades como dormir, sentarse, acostarse y 
ver la televisión, así como otras formas de entretenimiento ante una pantaIla. Desde el punto de vista operativo, el comportamiento sedentario incluye actividades con un gasto de energía que varía de 1 a 1.5 equivalentes metabólicos (MET). Un MET es el costo energético de descansar tumbado, a menudo definido en términos de consumo de oxígeno en $3.5 \mathrm{ml} . \mathrm{kg}$.min-1. La actividad física leve, a menudo asociada erróneamente con el comportamiento sedentario, es de hecho un tipo distinto de actividad, que implica un gasto de energía que oscila entre 1,6 y 2,9 MET. Esto corresponde a actividades como caminar despacio, escribir sentado, cocinar o fregar platos (Pate, O’Neill, \& Lobelo, 2008).

Con el fin de evaluar la actividad física de niños y adolescentes, se hace generalmente referencia a las recomendaciones de la Organización Mundial de la Salud (WHO, 2010). La idea es proporcionar puntos de referencia claros para una actividad física diaria beneficiosa para la salud. En otras palabras, definir el nivel mínimo de AF diaria que se debe practicar para garantizar una buena salud. Estas recomendaciones se formulan en términos de actividad física de moderada a vigorosa, que corresponde a un umbral de actividad de intensidad suficiente ( $>3 \mathrm{MET}$ ) para iniciar una serie de mecanismos fisiológicos de nuestro cuerpo: hablamos entonces de actividad física moderada a vigorosa. (AFMV o MVPA en inglés). La actividad física ligera no entra en la AFMV, lo cual explica que la AFMV y la vida sedentaria no son vasos comunicantes: eliminar el tiempo sedentario no se traduce necesariamente en un aumento de la AFMV ya que el nuevo comportamiento adoptado puede ser una actividad física ligera.

A continuación, se enumeran las principales recomendaciones para niños y adolescentes:

Para los niños y jóvenes en este grupo de edad, la actividad física incluye juegos, deportes, viajes, actividades recreativas, educación física o ejercicio planificado en la familia, la escuela o el entorno comunitario. Para mejorar la resistencia cardiorrespiratoria, la forma muscular y la condición ósea y reducir el riesgo de enfermedades no contagiosas, se recomienda lo siguiente:

Los niños y jóvenes de 5 a 17 años deben realizar al menos 60 minutos diarios de actividad física moderada a vigorosa.

1. La práctica de una actividad física durante más de 60 minutos al día proporcionará beneficios de salud adicionales.

2. La actividad física diaria debería ser esencialmente una actividad de resistencia. Las actividades de intensidad vigorosa, en particular aquellas que fortalecen el sistema muscular y la condición ósea, deberían practicarse al menos tres veces por semana (WHO, 2010).

Así pues, el punto de referencia para definir si un niño se considera suficientemente activo es 60 minutos de actividad física diaria. El término "inactivo" o "inactividad física" define un nivel insuficiente de actividad física de intensidad moderada a vigorosa (AFMV), es decir, por debajo del umbral recomendado. 


\section{Niveles de actividad $y$ sedentarismo para una jornada: Actividad física e inactividad.}

A nivel mundial, el 80,3\% de los adolescentes no cumple con las recomendaciones de AFMV. Los niños son un poco más activos que las niñas (Hallal et al., 2012). Para los niños es difícil encontrar valores normalizados, porque las variaciones metodológicas relacionadas con la medición de la actividad física llevan a resultados muy variables (Guinhouya, Samouda, \& De Beaufort, 2013).

En Francia, el estudio ESTEBAN (Santé Publique France, 2015), que es una referencia en esta materia, indica que el $72 \%$ de los niños y el $82 \%$ de las niñas no cumplen con las recomendaciones, una cifra bastante cercana a los valores mundiales.

Los estudios que realizamos localmente, basados en mediciones directas por acelerometría, indican que el $46 \%$ de los niños (Bernal et al., 2017) y el 83\% de los adolescentes (Aibar, Bois, Generelo, Zaragoza, \& Paillard, 2012) no llegan a las recomendaciones y, por lo tanto, se consideran insuficientemente activos. Si observamos el nivel medio de AFMV, es de $65 \mathrm{~min} /$ día y 43 min/día respectivamente para los niños y adolescentes de estos estudios (Aibar et al., 2012; Bernal et al., 2017). El fuerte y rápido descenso de los niveles de AFMV entre la infancia y la adolescencia está bien documentado y anima a llevar a cabo acciones de promoción desde una edad temprana.

\section{Sedentarismo}

Los trabajos sobre el sedentarismo son más difíciles de sintetizar, ya que los indicadores varían de un estudio a otro. Algunos estiman el tiempo pasado ante a las pantallas; otros el tiempo pasado viendo la televisión o el tiempo sentado, y otros miden incluso el tiempo sedentario total evaluado por acelerometría.

A nivel mundial, los datos disponibles indican que alrededor del $42 \%$ de los jóvenes de entre 15 y 29 años de edad reconocen pasar 4 horas al día o más sentados (Hallal et al., 2012). En Francia, el estudio ESTEBAN se centró en el tiempo pasado frente a una pantalla (TV, videojuegos, trabajo en el ordenador). Revela que casi el 50\% de los niños de 6 a 10 años, el 70\% de los de 11 a 14 años, el $71 \%$ de las niñas y el $87 \%$ de los de 15 a 17 años pasan 3 horas al día o más frente a una pantalla. En 10 años, este tiempo medio aumentó en 30 minutos en la franja de 6-10 años; 1 hora y 15 minutos en la de 11-14 años y 2 horas en la de 15-17 años (Santé Publique France, 2015).

Los estudios realizados a nivel local se refieren al tiempo sedentario total, es decir, el tiempo que se pasa sentado o de pie con muy poco gasto de energía. Estas mediciones realizadas por acelerometría indican que los niños dedican un promedio de 10 horas al día a actividades sedentarias, aproximadamente el 79\% del tiempo (Bernal et al., 2017). En adolescentes, los valores observados indican una media de 9 horas y 30 minutos de tiempo sedentario diario (Aibar et al., 2015). 


\section{Niveles de actividad y de sedentarismo observados en el horario escolar:}

Los estudios que muestran los niveles de actividad física y sedentarismo en el horario escolar son escasos, por lo que presentaremos los resultados de nuestro trabajo. Existen recomendaciones específicas para la AFMV realizada en el ámbito escolar. Se sugiere que el 50\% de la AFMV diaria se lleve a cabo en el entorno escolar (Pate et al., 2006), para facilitar el cumplimiento de los 60 minutos diarios recomendados.

Los datos que hemos observado en niños de Primaria indican que solamente el $38 \%$ de la AFMV diaria, aproximadamente 25 minutos, se realiza en el ámbito escolar (Bernal et al., 2017). Los valores observados en los adolescentes son mejores, ya que aproximadamente el $47.5 \%$ de su AFMV diaria se realiza en la escuela.

En cuanto al tiempo sedentario acumulado durante el horario escolar, sólo tenemos datos sobre niños de Primaria. Los resultados indican que aproximadamente el $81 \%$ del tiempo escolar de los niños es sedentario, es decir, 4 horas y 10 minutos de tiempo sedentario de las 5 horas y 30 minutos de tiempo escolar teórico (excepto la pausa de mediodía).

De este modo, el sistema escolar no consigue, por un lado, que los jóvenes sean lo suficientemente activos ya que rara vez se alcanza el 50\% de la AFMV diaria en el horario escolar. Y, por otro lado, genera una cantidad significativa de tiempo sedentario. Por lo tanto, parece urgente una toma de conciencia del papel central del sistema escolar en la promoción de la actividad física y en la lucha contra la vida sedentaria. Modificar la organización de la jornada escolar, un cambio de prácticas de enseñanza que lleve a clases más activas, así como un aumento en el volumen de las clases de Educación Física son vías que hay que explorar con urgencia.

\section{El contexto específico de las clases de Educación Física:}

Por definición, la clase de Educación Física es el contexto ideal para acumular un máximo de AFMV. Se recomienda que el 50\% del tiempo de la clase de Educación Física se realice en AFMV. Los datos disponibles indican que este objetivo rara vez se alcanza. Por ejemplo, un estudio suizo (Aibar \& Chanal, 2015) sobre más de 1.200 alumnos (de 10 a 12 años) revela que sólo alrededor del 25\% del tiempo de la sesión transcurre en AFMV y alrededor del 40\% en tiempo sedentario. Durante el tiempo restante, el alumnado realiza una actividad física ligera. Por supuesto, es probable que estas cifras varíen considerablemente según el tipo de sesión, las actividades practicadas o la distancia entre la escuela y el lugar de práctica. En todo caso, la brecha entre la actividad real de los estudiantes y las recomendaciones parece todavía muy importante.

\section{Conclusión:}

Los datos presentados revelan niveles de AFMV globalmente preocupantes para niños y adolescentes. Un bajo nivel de actividad física durante estas edades hace prever una actividad física insuficiente en la edad adulta. Sin embargo, la 
actividad física es un factor de prevención y protección contra la mayoría de las enfermedades no transmisibles, como la diabetes, la hipertensión, las enfermedades cardiovasculares o diferentes tipos de cáncer (INSERM, 2019; Warburton, Nicol, \& Bredin, 2006).

Los datos presentados muestran que ni en el contexto escolar general ni en el más específico de la Educación Física se alcanzan los niveles necesarios para mantener una buena salud. Ahora bien, la escuela es, para el poder público, un medio ideal a través del cual podría promover entre los más jóvenes hábitos de vida saludables. El estudio realizado constata que queda mucho por hacer, a pesar de la gran importancia que tiene el tema para la salud de las generaciones futuras y la reducción del gasto sanitario que podría generar. Por consiguiente, promover la actividad física y reducir el sedentarismo en entornos escolares debería ser un objetivo prioritario para las futuras políticas de salud pública.

\section{Bibliografía:}

Aibar, A., Bois, J. E., Generelo, E., Zaragoza, J., \& Paillard, T. (2012). A cross-cultural study of adolescents' physical activity levels in France and Spain. European Journal of Sport Science, 13(5), 551-558. doi: 10.1080/17461391.2012.746733

Aibar, A., \& Chanal, J. (2015). Physical education: The effect of epoch lengths on children's physical activity in a structured context. PLOS ONE, 10(4). doi: 10.1371/journal.pone.0121238

Aibar, A., Bois, J. E., Zaragoza, J., Bru, N., Paillard, T., \&, Generelo, E. (2015). Adolescents' Sedentary Behaviors in Two European Cities. Research quarterly for exercise and sport, 86(3), 233-243. doi: 10.1080/02701367.2015.1039891

Bernal, C., Lhuisset, L., Fabre, N., Chanal, J., Ruelloux, J., \& Bois, J. (2017). Influence de l'activité physique et des habiletés motrices sur les capacités attentionnelles et la réussite scolaire chez des enfants d'école primaire. Paper presented at the Congrès International de l'Association des Chercheurs en Activités Physiques et Sportives (ACAPS), Dijon.

Caspersen, C. J., Powell, K. E., \& Christenson, G. (1985). Physical activity, exercise and physical fitness: definitions and distinctions for health-related research. Public Health Reports, 100(2), 126-131.

Guinhouya, B. C., Samouda, H., \& De Beaufort, C. (2013). Level of physical activity among children and adolescents in Europe: A review of physical activity assessed objectively by accelerometry. Public Health, 127(4), 301-311. doi: 10.1016/j.puhe.2013.01.020

Hallal, P. C., Andersen, L. B., Bull, F. C., Guthold, R., Haskell, W., \& Ekelund, U. (2012). Global physical activity levels: Surveillance progress, pitfalls, and prospects. The Lancet, 380(9838), 247-257. doi: 10.1016/s0140-6736(12)60646-1

INSERM. (2019). Activité physique. Prévention et traitement des maladies chroniques. In E. Sciences (Ed.), (pp. 824).

Pate, R. R., Davis, M. G., Robinson, T. N., Stone, E. J., McKenzie, T. L., \& Young, J. C. (2006). Promoting physical activity in children and youth: A leadership role for schools - A scientific statement from the American Heart Association Council on Nutrition, Physical Activity, and Metabolism (Physical Activity Committee) in collaboration with the Councils on Cardiovascular Disease in the Young and Cardiovascular Nursing. Circulation, 114(11), 1214-1224. doi: 10.1161/circulationaha.106.177052

Pate, R. R., O'Neill, J. R., \& Lobelo, F. (2008). The evolving definition of "sedentary". Exercise and Sport Sciences Reviews, 36(4), 173-178. doi: 10.1097/JES.0b013e3181877d1a

Santé Publique France. (2015). Étude de santé sur l'environnement, la biosurveillance, l'activité physique et la nutrition (Esteban) 2014-2016. Volet nutrition. Chapitre Activité physique et sédentarité.

Warburton, D. E. R., Nicol, C. W., \& Bredin, S. S. D. (2006). Health benefits of physical activity: The evidence. CMAJ, 174(6), 801-809. doi: 10.1503/cmaj.051351

WHO (2010). Global Recommendations on Physical activity for Health. Geneva: World Health Organization. 


\title{
La escuela y la promoción de la salud del alumnado
}

\author{
Carole Sève ${ }^{1}$ \\ ${ }^{1}$ Inspectora General de Educación Nacional, \\ Ministerio de Educación Nacional, París.
}

\section{Resumen:}

El propósito de esta presentación es examinar la forma en que la institución escolar ha abordado los temas de promoción de la salud de los escolares. Se centra en la enseñanza de la Educación Física y señala que, mientras que la salud se incluye explícitamente en los retos de la asignatura, en la práctica los profesores de Educación Física dejan a menudo la preocupación por la salud en un segundo plano. La asignatura de Educación Física por sí sola no puede alcanzar los retos de salud establecidos por la institución escolar: es un asunto colectivo que debe involucrar a toda la comunidad educativa.

\section{La escuela y la promoción de la salud del alumnado:}

Promover la salud en la escuela es un reto importante en la lucha contra las desigualdades escolares (Pinel-Jacquemin, 2016). De hecho, varios estudios muestran la relación entre la salud de los estudiantes y su éxito académico. La Ley de julio de 2013 sobre la Refundación de la Escuela de la República describe siete ejes principales para la promoción de la salud en la escuela: establecer un ambiente escolar saludable, la implementación de programas de educación en salud, la participación en la política de prevención sanitaria, llevar a cabo controles de salud, la detección de problemas de salud, el apoyo al alumnado y la participación en la vigilancia epidemiológica. Estos ejes cubren así un campo muy amplio que va desde la protección hasta la prevención pasando por la educación. Varias circulares describen diferentes iniciativas para hacer realidad estos ejes. Por ejemplo, el Itinerario de Educación para la Salud (circular de enero de 2016) tiene como objetivo reunir y ordenar las distintas acciones de promoción de la salud llevadas a cabo en un centro educativo, para luchar contra la ineficacia de la multiplicación de acciones aisladas en salud mencionada por Broussouloux y Lamoureux (2007); los Comités de Educación para la Salud y la Ciudadanía (circular de agosto de 2016) se encargan de implementar una educación para la salud en las escuelas mediante la participación de diferentes socios educativos.

El enfoque escolar de la salud por parte de la institución escolar, se basa en un concepto global de la salud (en referencia a la definición de salud de la OMS) que integra componentes fisiológicos, psicológicos y sociales: relaciona salud, responsabilidad y ciudadanía. El programa de educación para la 
salud enfatiza la importancia de la participación de los estudiantes y su actitud reflexiva. Se trata de que los estudiantes tomen conciencia de ciertos conocimientos y experiencias que les permitan cuidarse a sí mismos y a los demás, evitando comportamientos de riesgo.

Si la institución escolar insiste en el compromiso de toda la comunidad educativa para hacerse cargo de la salud del alumnado, en la práctica afecta esencialmente a tres categorías profesionales: los enfermeros escolares, que aplican la política de salud del Ministerio de Educación Nacional y que, a menudo, se involucran en acciones de educación para la salud; los psicólogos escolares, que cuidan del sufrimiento psicológico de los niños; y los docentes, cuya participación en la educación para la salud está incluida en su cometido profesional. Sin embargo, las actitudes de los docentes hacia la educación para la salud son muy diversas. En un estudio sobre la participación de maestros de preescolar en un programa de educación para la salud, Gaborit (2015) identifica cuatro perfiles: Los "docentes buenos alumnos" (que aplican el programa); los "docentes desbordados" (que no tienen tiempo para realizar el programa porque tienen otras prioridades); los "maestros refractarios" (que rechazan el programa porque está en contradicción con sus propios valores); y los "profesores distantes" (que no están interesados en el programa).

Además, cuando hablamos de educación para la salud en la escuela, dos asignaturas escolares son a menudo subrayadas: Las Ciencias Sociales y Naturales (debido a los temas de su programa relacionados con la salud) y la Educación Física. Esta referencia a la Educación Física se basa en representaciones sociales ("el deporte es salud"), en el conocimiento científico (el vínculo entre la actividad física y la salud) y en los programas de esta asignatura en los que se presentan explícitamente problemas de salud. Para los ciclos 2, 3 y 4 (ciclos de Primaria), una de las habilidades generales en los programas de Educación Física es "Aprender a mantener la salud a través de la actividad física regular". La forma en que esta habilidad se desarrolla en los tres ciclos interpreta la salud como la gestión de las capacidades físicas de cada cual. En la escuela secundaria, un objetivo general de la Educación Física es "Construir una salud duradera" e incluye en su presentación aspectos fisiológicos, psicológicos y sociales. Respecto a las concepciones de la salud en la Educación Física, Moralès y Travaillot (2015) distinguen dos corrientes principales: (a) una corriente denominada hedonista en la que se hace hincapié en el compromiso físico, el bienestar y el placer; y (b) una corriente que llaman apropiativa en la cual el enfoque está en la adquisición de habilidades y conocimientos específicos (en y para la salud). Estas dos corrientes expresan dos enfoques diferentes de la salud. Sin embargo, en la práctica docente de los profesores de Educación Física, parece que esta preocupación por la salud del alumnado pasa en un segundo plano en comparación con otras cuestiones. De hecho, los estudios sobre la actividad de los profesores de Educación Física destacan a menudo las preocupaciones relacionadas con la disciplina, la participación activa de los estudiantes, el aprendizaje, el desarrollo de su autonomía... pero rara vez con la salud. Además, los profesores de Educación Física definen la educación para la salud de una manera diversa y 
con frecuencia ambigua (Loison, 2015). Además, parece que este reto de la salud asociado con la Educación Física se centra más en la propia asignatura que en establecer estrategias de enseñanza reales. La integración de los retos de salud en la práctica docente de los profesores de Educación Física sigue siendo un desafío y debe organizarse en torno a varios principios: una definición compartida de salud; una concepción de la salud que tenga en cuenta los perfiles específicos de los estudiantes para evitar un enfoque normalizante y moralizante; la introducción de contenidos didácticos específicos de salud según las necesidades de los alumnos; la propuesta de actividades que favorezcan el compromiso físico del alumnado; una organización de la práctica para ofrecer al alumnado experiencias de bienestar perceptibles a corto y medio plazo. Esto sabiendo que el volumen semanal de Educación Física es, según los cursos, entre 2 y 4 horas, y que persigue otros objetivos educativos. La asignatura de Educación Física, por sí misma, no puede lograr los objetivos de educación para la salud establecidos por la escuela. Promover la salud del alumnado en la escuela es un asunto colectivo que debe involucrar a toda la comunidad educativa, ser sostenido en el tiempo y proponer acciones complementarias y coherentes en diferentes niveles.

\section{Bibliografía:}

Broussouloux S., \& Lamoureux, P. (2007). Le développement de l'éducation à la santé à l'école. Situations de la France et du Québec. Les Tribunes de la santé 3(16), 61-68.

Gaborit, E. (2015). Sanitarisation de l'école et scolarisation de la santé. Socioéthnographie de la mise en oeuvre et appropriation du programme "Bien manger et bouger à la maternelle" en Haute Garonne et en Ariège. Thèse, Faculté des Sciences du Sport et du Mouvement Humain, Université Toulouse III, Toulouse. Co-dirigée par Nadine Haschar-Noé et Gérard Neyrand.

Loison D. (2015) Quels savoirs enseignés en éducation à la santé dans les séances d'EPS ? Éducation, Santé, Sociétés, 2(1), 13-31.

Morales Y., \& Travaillot Y. (2015). Débats et controverses autour des programmes d'EPS. Revue Education, Santé, Société, 2, 95-110.

Pinel-Jacquemin S. (2016). Bien-être des élèves à l'école et promotion de leur santé. Rapport commandé par le Cnesco. https://www.cnesco.fr/fr/qualite-de-vie-a-lecole/ 


\section{La promoción de la actividad física para la salud en Educación Física en España}

José Antonio Julián ${ }^{1-2}$ y Alberto Aibar ${ }^{2-3}$

${ }^{1}$ Facultad de Ciencias Sociales y Humanas. Universidad de Zaragoza.

${ }^{2}$ Grupo de Investigación EFYPAF. Universidad de Zaragoza.

${ }^{3}$ Facultad de Ciencias Humanas y de la Educación. Universidad de Zaragoza.

\section{Resumen}

La legislación española vigente muestra la obligación de las administraciones educativas de promover la práctica de actividad física durante la jornada escolar. Son numerosas las iniciativas que por parte de los diferentes ministerios y de las Comunidades Autónomas se han realizado para nutrir al profesorado y a las familias de los centros educativos de recursos con los que llevar a cabo esa promoción.

Mostramos ocho decisiones que el profesorado y los centros educativos pueden tomar de cara a implicar al mayor número de agentes posible para generar ambientes saludables en sus contextos. Se finaliza haciendo hincapié en la responsabilidad de la administración para liderar iniciativas basadas en la evidencia científica.

\section{Introducción:}

La relación entre educación y salud plantea un potencial de influencia bidireccional tan importante que su presencia no puede ser obviada a nivel escolar. Ambos conceptos deben de ir de la mano en el proceso de construcción social de los futuros ciudadanos que se encuentran actualmente en las aulas de la educación obligatoria. Resulta ampliamente conocido y aceptado el hecho de que los estudiantes más sanos aprenden mejor y que aquellos adultos más educados resultan sujetos más saludables (Kolbe, 2019). Por ello, resulta fundamental la promoción de asociaciones colaborativas entre sistemas que mejoren los niveles de salud y de educación de la sociedad en su conjunto. La actividad física (AF) y su promoción con el objeto de crear ciudadanos alfabetizados físicamente se vuelve un elemento clave de esa relación potencial entre salud y educación. El modelo conceptual WSCC de la ASCD (The Whole School Whole Community, Whole Child) nos sirve como aproximación teórica de referencia que aúna los objetivos comunes de ambos sectores y que sitúa la actividad física y la Educación Física como un componente esencial a trabajar dentro de la comunidad. 


\section{El papel de la escuela en la promoción de la actividad física en España:}

La escuela, y más concretamente la Educación Física (EF), juega un papel fundamental en la construcción de un estilo de vida activo y saludable (Abarca-Sos, Murillo, Julián, Zaragoza, \& Generelo, 2015). Modelos como el Programa Intregral de actividad física escolar (CSPAP; National Association for Sport and Physical Education, 2008) sitúan a la EF como una de las piezas clave dentro de las opciones de práctica que ofrece el día a día escolar.

Esta situación se ve reforzada por la legislación española vigente (Ley Orgánica 8/2013), la cual reconoce la obligación de las administraciones, y en consecuencia de los centros educativos, de promover la práctica de actividad física durante la jornada escolar. Para ello, existen multitud de iniciativas extra-curriculares y materiales didácticos curriculares generados por la administración para acompañar y ayudar a la comunidad educativa en general, y a los maestros en particular, en su trabajo de promoción de la actividad física (por ejemplo, la estrategia nacional NAOS, de Nutrición, Actividad Física y Prevención de la Obesidad).

\section{Decisiones para la promoción de la actividad física en la escuela:}

Según nuestro conocimiento de la realidad del contexto educativo cuando hay una preocupación por potenciar un modo de vida activo y saludable entre el alumnado, el profesorado y los centros educativos pueden adoptar una serie de decisiones de cara a implicar al mayor número de agentes posible y así generar ambientes más saludables. Son las siguientes:

1. La organización de la Educación Física.

2. Optimizar la metodología de las actividades.

3. Desarrollar acciones para integrar los aprendizajes de la "gestión de la vida activa".

4. Conectar el contexto escolar con la realidad social.

5. Desarrollar proyectos interdisciplinares.

6. Desarrollar un proyecto global de promoción de la AF y la salud.

7. Proyectos globales de promoción de la AF coordinados entre centros.

8. Institucionalizar y evaluar las acciones que se realizan desde el contexto escolar.

Explicaremos brevemente cada una de estas decisiones algunas recogidas ya en Julián, Abarca-Sos, Zaragoza, \& Aibar (2016).

1) La organización de la Educación Física. En base a la propuesta de los dominios de acción motriz realizada por Parlebas (2006) y a otros trabajos de innovación educativa que se han ido realizando en nuestro contexto, existen 5 bloques de contenido con esta denominación: Bloque 1. Ac- 
ciones motrices individuales; Bloque 2. Acciones motrices de oposición; Bloque 3. Acciones motrices de cooperación y colaboración-oposición; Bloque 4. Acciones motrices en el medio natural; Bloque 5. Acciones motrices con intenciones artísticas o expresivas. De esta manera se invita al profesorado a que su alumnado reciba a lo largo de la etapa un tratamiento adecuado del área de EF, a través de un trabajo sistemático (todos los cursos), equilibrado (parecido número de sesiones en los cinco primeros bloques) y contextualizado (ajustado a las posibilidades del centro y del entorno) de los diferentes tipos de experiencias motrices.

2) Optimizar la metodología de las actividades. Se hace necesario proporcionar experiencias satisfactorias de aprendizaje con el desarrollo de climas motivaciones óptimos en todas las unidades didácticas (Julián, 2012).

3) Desarrollar acciones para integrar los aprendizajes de la "gestión de la vida activa". Los aprendizajes relacionados con la pedagogía a través/ desde las conductas motrices y los aprendizajes transversales se agrupan en un bloque específico. Este bloque, denominado "Gestión de la vida activa y valores" muestra diferentes aprendizajes importantes para el área como son: las reglas y principios para actuar; la actividad física, hábitos posturales, higiénicos y alimenticios; los efectos fisiológicos de la realización de actividad física relacionados con la salud; la aceptación de las diferentes realidades corporales; el reconocimiento del patrimonio cultural; la construcción de la vida activa; y el desarrollo de valores individuales, sociales y medio ambientales. La idea que debe predominar en el tratamiento de este bloque, es que debe desarrollarse globalmente con las situaciones de aprendizaje que el docente programará de los otros cinco bloques o mediante acciones específicas durante el curso escolar.

4) Conectar el contexto escolar con la realidad social. Debemos aprovechar los eventos sociales y culturales del contexto próximo relacionados con la AF para ir disponiendo nuestras unidades didácticas de manera que nos sirvan para poder poner en acción todos los aprendizajes que se han desarrollado durante la misma. De esta forma estaremos dando mayor funcionalidad a los aprendizajes demandados a los estudiantes.

5) Desarrollar proyectos interdisciplinares. La EF tiene un gran potencial para la movilización de aprendizajes escolares, ya que las actividades propuestas al alumnado son una situación competencial por sí mismas; movilizan todos los recursos disponibles (saberes, actitudes, conocimientos, habilidades, etc.) en unas condiciones que se conectan con la vida real del alumnado. Los estudiantes identifican claramente en su día a día la natación, la carrera de larga duración, el tenis, la lucha, el baloncesto, el voleibol, los desafíos cooperativos, la orientación, la BTT, las danzas tradicionales, la expresión corporal, etc., lo que provoca que 
tenga un potencial globalizador y aglutinador importante en el ámbito educativo (Julián, Ibor, Aibar, \& Aguareles, 2017). Para profundizar en esta idea recomendamos la propuesta de Julián et al. (2017).

6) Desarrollar un proyecto global de promoción de la AF y la salud. Es interesante desarrollar proyectos globales desde el marco establecido por las escuelas promotoras de salud para conectar los diferentes agentes y actores que influyen en un contexto educativo. Como sabemos, cuantas más conexiones y más espacios y tiempos estén intencionalmente enriquecidos más posibilidades hay de desarrollar un ambiente saludable (Sevil, García-González, Generelo, \& Aibar, 2019).

7) Proyectos globales de promoción de la AF coordinados entre centros. Es un reto maravilloso que diferentes escuelas con proyectos de promoción de la AF similares se encuentren en varios momentos temporales durante una etapa educativa, para potenciar interacciones con la AF como protagonista (Navarro et al., 2017).

Las decisiones narradas anteriormente, se presentan en un nivel creciente de dificultad debido a los agentes y actores que hay que movilizar e implicar para poder llevarlas a término. A su vez, en cada decisión se podrían determinar niveles de calidad diferentes de cara a optimizarlas.

\section{Conclusión:}

La última decisión es institucionalizar y evaluar las acciones que se realizan desde el contexto escolar. Preguntas como: ¿qué funciona en la promoción de la AF desde los centros escolares?, ¿qué condiciones necesitan los centros para desarrolla proyectos trasformadores? o ¿qué estrategias son más efectivas? son cuestiones que la administración debe responder en colaboración con investigadores y centros educativos. La promoción de la AF exige la necesidad de un liderazgo político claro que encamine a las administraciones educativas y sanitarias a constituirse como instituciones realmente claves en la coordinación, orientación y evaluación de proyectos basados en la evidencia científica que retroalimenten a su vez los procesos de promoción de conductas saludables como la AF. 


\section{Bibliografía:}

ASCD: www.ascd.org/learningandhealth

Abarca-Sos, A., Murillo, B., Julián, J. A., Zaragoza, J., \& Generelo, E. (2015). La Educación Física: ¿Una oportunidad para la promoción de la actividad física? Retos, 28, 155-159.

Estrategia nacional española NAOS. http://www.aecosan.msssi.gob.es/AECOSAN/web/ nutricion/seccion/estrategia_naos.htm

Julián, J. A. (2012). Motivación e intervención docente en la clase de educación física. Tándem. Didáctica de la Educación Física, 40, 7-17.

Julián, J. A., Abarca-Sos, A., Zaragoza, J., \& Aibar, A. (2016). Análisis crítico de la propuesta del currículum básico de la LOMCE para la asignatura de Educación Física. Acciones derivadas en la Comunidad Autónoma de Aragón y propuestas de futuro. Retos, 29, 173-181.

Julián, J. A., Ibor, E., Aibar, A., \& Aguareles, I. (2017). Educación física, motor de proyectos. Tándem. Didáctica de la Educación Física, 56, 7-15.

Julián, J. A., Zaragoza, J., Abarca-Sos, A., Aibar, A., Tena, I., Ibor, E.,..., \& Tartaj, N. (2017). Los proyectos interdisciplinares en la formación inicial del profesorado de educación física. Aula de Innovación Educativa, 266, 12-16.

Kolbe, L. J. (2019). School health as a strategy to improve both public health and education. Annual Review of Public Health, 40(3), 1-3. https://doi.org/10.1146/annurevpublhealth-040218-043727

Ley Orgánica 8/2013, de 9 de diciembre, para la Mejora de la Calidad Educativa (LOMCE), Boletín Oficial del Estado (BOE). 10 de diciembre de 2013.

National Association for Sport and Physical Education (2008). Comprehensive school physical activity programs. Reston, VA: Author. Available at https://www.cdc.gov/healthyschools/ physicalactivity/cspap.htm

Navarro, A., Martín, D., García, C., Jofre, E., Hernández, P., \& Gómez, D. (2017). Proyecto Amigos Activos. Tándem. Didáctica de la Educación Física, 55, 59-66.

Sevil, J., García-González, L. Generelo, E., \& Aibar, A. (2019). Can high schools be an effective setting to promote healthy lifestyles? Effects of a multiple behavior change intervention in adolescents. Journal of Adolescent Health, 64(4), 478-486. doi: 10.1016/j.jadohealth.2018.09.027

Parlebas, P. (2006). L'action motrice, fer de lance de l'éducation physique. Les Cahiers EPS de l'Académie de Nantes, 34, 5-9. 


\section{Curso teórico puesto en práctica en el Collège de Lourre Barrousse.}

Ludovic Dolce $^{1}$ y Magalie Annecy ${ }^{1}$

Collège Loures Barousse, EPS

\section{Situación al inicio de la acción en 2012:}

Durante el año escolar 2012-2013 se valoró en el Collège Barousse la posibilidad de participar en el proyecto experimental "Aprender de manera diferente" -como continuación del experimento nacional "Clases por la mañana, Deporte por la tarde", que estaba ya en su recta final- con el objetivo de permitir a los alumnos de $6^{\circ}$ curso (11-12 años) una buena integración en el ciclo secundario trabajando el sentido de la escuela y los métodos de aprendizaje.

\section{1 - Objetivos perseguidos}

La puesta en marcha de este proyecto experimental apunta a varios objetivos:

1. Ofrecer a los alumnos con dificultades académicas o con necesidades educativas específicas una estructura pequeña, dinámica e innovadora con un número de integrantes por clase óptimo (unos 20 alumnos por clase).

2. Establecer ritmos escolares propicios para el aprendizaje.

3. Provocar en la escuela una reflexión sobre el aprendizaje de los alumnos.

4. Favorecer el trabajo en equipo.

5. Número de alumnos y nivel(es) concernidos: este nuevo proyecto experimental afecta a los alumnos de sexto curso (11-12 años), es decir, un promedio de 40 alumnos.

\section{2 - Descripción}

Se conserva el formato actual de los horarios, basado en una adaptación del ritmo escolar al ritmo diario y semanal más favorable para el aprendizaje, y el trabajo se orienta de una manera más motivadora y metacognitiva. Sabemos que el aprendizaje escolar exige un esfuerzo a los estudiantes, 
de ahí el trabajo de motivación para dar sentido al aprendizaje. También sabemos que, a menudo, los estudiantes carecen de métodos de aprendizaje.

\section{3 - Métodos de implementación}

Los tiempos generados en el marco del proyecto están especialmente dedicados a trabajar en estos dos ejes: motivación y meta-aprendizaje para que los estudiantes tomen conciencia de que los conocimientos y habilidades adquiridos durante la semana se pueden transferir a las actividades prácticas el jueves y el viernes por la tarde (horario del proyecto).

Los jueves, preferentemente a actividades educativas y culturales, y los viernes por la tarde a actividades deportivas. El trabajo en equipo interdisciplinario es esencial para alimentar esta práctica.

"Todos los viernes están reservados a salidas para realizar actividades físicas al aire libre, donde la energía es el pilar central de los circuitos pedestres. Esta actividad se organiza con las 2 clases todos los viernes de 14h a 17h. Lucha contra el sedentarismo, la obesidad... Las habilidades sociales también están en el centro de estas salidas deportivas".

\section{4 - Recursos utilizados}

Siete horas semanales suplementarias permiten a los profesores de cada asignatura poner en práctica los conocimientos teóricos, de manera transversal, en colaboración con el profesorado de Educación Física.

Un presupuesto de 3.000 euros al año permite prever los desplazamientos y la participación de personas ajenas al centro (el proyecto experimental "Clases por la mañana, Deporte por la tarde" tenía una financiación de 500 euros).

\section{5 - Asociación y contenido de la asociación}

Trabajar en colaboración con los ayuntamientos y la mancomunidad de Barousse debido a la implementación del proyecto en numerosos sitios históricos y naturales próximos al centro escolar.

\section{6 - Evaluación}

Modalidades de seguimiento y evaluación de la acción (autoevaluación, evaluación interna, externa): Un comité directivo se reúne mensualmente para revisar la organización del programa y sus mejoras.

Validación de la etapa 2 de las bases de conocimientos comunes para alumnos que no la habían superado en CM2 (10-11 años).

Tres recursos o puntos de apoyo que os permitieron progresar. 
- Patrimonio histórico y natural próximo al centro escolar.

- El trabajo en equipo del profesorado.

- El éxito del primer proyecto "Clases por la mañana, Deporte por la tarde".

Dos posibles dificultades encontradas.

- La adaptación de los horarios semanales.

- Los medios financieros insuficientes para permitir las diferentes salidas.

\section{Efectos observados:}

- En los conocimientos del alumnado.

- Bienestar del alumnado de $6^{\circ}$ curso (11-12 años) en el centro escolar.

- Cohesión entre el alumnado.

- El alumnado con necesidades especiales se encuentra con menos dificultades. En el trabajo del profesorado.

- Desarrollo de la transdisciplinariedad.

- Adaptación de algunas prácticas a otros niveles en relación con el liderazgo y las relaciones profesionales.

- En la escuela / centro escolar.

- Refuerza su imagen innovadora en términos de aprendizaje general.

- En el entorno.

\section{2. ¿Y en qué punto estamos hoy, en 2018?}

Tuvimos que hacer evolucionar nuestro sistema (que fue originalmente experimental... durante 3 años). Hoy, APRENDER DE MANERA DIFERENTE es un sistema que sigue vigente en nuestro centro, pero sólo gracias a la motivación del personal que está muy comprometido con este sistema. De hecho, desde el final del período de experimentación, ya no tenemos ningún medio (financiero, humano) asignado a este sistema. Tenemos que arreglarnos con los recursos propios del centro.

Unas asignaturas han cedido horas para poder funcionar, las salidas son a menudo de pago y requieren un esfuerzo por parte del centro.

El alumnado de $6^{\circ}$ curso (11-12 años) realiza salidas para actividades al aire libre cada 3 semanas. Se alternan con las clases de Valores y Ciudadanía, el Premio Literario y la Ayuda Personalizada.

Las salidas para actividades al aire libre y Aprender de manera diferente son las siguientes: usamos el tejido local... 


\begin{tabular}{|c|c|c|}
\hline & $\begin{array}{l}\text { Asignaturas relacionadas con } \\
\text { EF }\end{array}$ & Trabajos durante la salida \\
\hline 1 & Ciencias Sociales y Naturales & $\begin{array}{l}\text { Clasificación de plantas durante un ex- } \\
\text { cursión a Sarp ( } 20 \text { minutos a pie desde } \\
\text { el centro). 3h }\end{array}$ \\
\hline 2 & Geografía e Historia & $\begin{array}{l}\text { Análisis del paisaje desde la cima del } \\
\text { Mont Bourg lzaourt }(700 \mathrm{~m}) .4 \mathrm{~h}\end{array}$ \\
\hline 3 & Valores y Ciudadanía & $\begin{array}{l}\text { En el Ayuntamiento de Loures Barousse } \\
\text { (10 min a pie). 3h }\end{array}$ \\
\hline 4 & Música & $\begin{array}{l}\text { Trabajo sobre el órgano de St Bertrand de } \\
\text { Comminges con el organista local: vamos } \\
\text { andando o en bicicleta de montaña según } \\
\text { el número de alumnos. } 3 \mathrm{~h}\end{array}$ \\
\hline 5 & Matemáticas & $\begin{array}{l}\text { Trabajo matemático sobre los "arcos" a } \\
\text { través de una carrera de orientación en la } \\
\text { ciudad medieval. } 3 \mathrm{~h}\end{array}$ \\
\hline 6 & Valores y Ciudadanía & $\begin{array}{l}\text { Excursión con raquetas de nieve en } \\
\text { Mourtis ( } 45 \text { min en bus). } 4 \mathrm{~h}\end{array}$ \\
\hline 7 & $\begin{array}{l}\text { Relación colegio / instituto con } \\
\text { los profesores de las escuelas } \\
\text { de la zona } \\
\text { - St Bertrand } \\
\text { - Loures } \\
\text { - Saléchan }\end{array}$ & $\begin{array}{l}\text { Trabajo multidisciplinar para una jornada: } \\
\text { Año par: trabajo de proporcionalidad } \\
+ \text { lengua con el libro "L'enfant océan ": } \\
\text { escalada + carrera de orientación. } \\
\text { Año impar: trabajo en las ruinas ro- } \\
\text { manas de St Bertrand de Comminges, } \\
\text { lengua, artes plásticas y matemáticas: } \\
\text { carrera de orientación + tiro con arco. }\end{array}$ \\
\hline
\end{tabular}

Nuestro alumnado aumenta constantemente en el Collège Barousse. Este año fue más difícil mantener este sistema porque hasta ahora solo teníamos dos grupos de $6^{\circ}$ curso (11-12 años), ipero este año tenemos tres! Pero nos las arreglamos, desafortunadamente eliminando salidas... 


\section{Ejemplo de innovación educativa. El trabajo colaborativo: efecto de un sistema de aprendizaje cooperativo para el bienestar psicosocial de los alumnos.}

Pascal Legrain $^{1}$ y Guillaume Escalie ${ }^{1}$ 1 UF STAPS, Université de Bordeaux

\section{Resumen:}

La reciente constitución de un ciclo CM1-CM2-6 ${ }^{\circ}$ curso (9-10 años/1011 años/11-12 años) exige un cambio en las prácticas de enseñanza para garantizar la continuidad del aprendizaje de los estudiantes entre la escuela primaria y el instituto. La sistematización de ciertos "formatos pedagógicos" de manera sincrónica (entre asignaturas) y diacrónica (a lo largo del ciclo) puede constituir un vector de estabilización del aprendizaje en la transición inter-grado que favorezca el bienestar psicosocial del alumnado.

En una tradición socio-constructivista de adquisiciones, este texto da cuenta de una investigación colaborativa a escala multidisciplinar cuyo objetivo es apoyar al profesorado de una futura red de escuelas e institutos basándose en un modelo común, en innovadores mecanismos de aprendizaje cooperativo y su puesta en práctica en clase. Desde un punto de vista metodológico, los datos cuantitativos para examinar los efectos de la implementación de estos sistemas en los aspectos psicosociales relacionados con el bienestar de los estudiantes se combinaron con datos cualitativos, que analizaron el impacto de la puesta en práctica de estos sistemas sobre la actividad del profesorado en clase.

Por un lado, los resultados muestran efectos significativos de este tipo de sistemas en la motivación personal de los estudiantes o en la menor incidencia de casos de ansiedad. Por otro, subrayan la necesidad de considerar la implementación de estos dispositivos en sus dimensiones tanto genéricas como disciplinarias. 


\section{Bibliografía:}

Aronson, E., \& Patnoe, S. (1997). The jigsaw classroom: Building cooperation in the classroom. New York: Addison-Wesley.

Cohen, E. G. (1994). Restructuring the classroom: Conditions for productive small groups. Review of Educational Research, 64, 1-35.

Gillet, N., Rosnet, E., \& Vallerand, R. J. (2008). Development of a scale to assess basic need satisfaction in the sport context. Canadian Journal of Behavioural Science, 40, 230-237.

Gillet, N., Vallerand, R. J., Amoura, S., \& Baldes, B. (2010). Influence of coaches' autonomy support on athletes' motivation and sport performance: A test of the hierarchical model of intrinsic and extrinsic motivation. Psychology of Sport and Exercise, 11, 155-161.

Johnson, D. W., \& Johnson, R. T. (2014). Cooperative learning in 21th century. Anales de Psicología, 30, 841-851.

Vallerand, R. J., Blais, M. R., Brière, N. M., \& Pelletier, L. G. (1989). Construction et validation de I'Échelle de Motivation en Éducation (EME). Revue canadienne des sciences du comportement, 21, 323-349. 


\section{Intervención escolar para promover la actividad física y disminuir el tiempo de sedentarismo en una población de niños de barrios desfavorecidos de 6 a 11 años}

Caroline Bernal', Nicolas Fabre', Léna Lhuisset ${ }^{1}$ y Julien Bois`'
'Université de Pau et des Pays de l'Adour
Laboratoire « Mouvement, Equilibre, Performance et Santé »

\section{Resumen:}

Con el advenimiento de la era tecnológica, estamos presenciando una disminución de los niveles de la actividad física (AF) y un aumento del tiempo sedentario (TS) de los niños. En dos escuelas primarias ubicadas en un barrio desfavorecido de Tarbes se comprobó que los niños pasan un promedio de 10 horas en una actitud sedentaria y que el $37 \%$ de los niños también están inactivos. A lo largo de 11 meses se llevó a cabo una intervención en las dos escuelas estudiadas. Se utilizaron diversas estrategias para promover la actividad física e involucrar al alumnado, sus familias, la escuela, la comunidad y las políticas públicas (Bronfenbrenner, 1977).

\section{Contexto:}

La actividad física (AF), definida como cualquier movimiento producido por los músculos esqueléticos que conlleva un aumento del gasto energético, tiene muchos beneficios para la salud física, mental y social de los niños (Grélot, 2016). También tendría repercusiones positivas en las capacidades cognitivas de los niños y, especialmente, en las de atención, inhibición y flexibilidad mental (Donnelly et al, 2016). La Organización Mundial de la Salud (OMS) recomienda que los niños practiquen 60 minutos de actividad física moderada y vigorosa al día, incluyendo 30 minutos en el horario escolar. Un estilo de vida sedentario se define como un conjunto de comportamientos que requieren un gasto energético muy bajo ( $<1,5 \mathrm{METs})$ en posición sentada y de reposo (Barnes et al., 2012). La OMS recomienda disminuir tanto como sea posible la acumulación de tiempo sedentario a lo largo del día.

\section{Estudio:}

Este estudio se lleva a cabo en dos escuelas primarias ubicadas en la "Red de Educación Prioritaria" de la ciudad de Tarbes: la Escuela Jean-Jacques Rousseau y la Escuela Jules Verne. En noviembre de 2016 y mayo de 2017 se llevaron a cabo mediciones iniciales para evaluar los niveles de activi- 
dad física y vida sedentaria de los niños, así como el desarrollo de sus principales capacidades de atención. Encontramos que el 37\% de los niños no cumplen con las recomendaciones de la OMS y que acumulan más de 10 horas de tiempo sedentario al día. El $80 \%$ del tiempo entre las $12 \mathrm{~h}$ y las $14 \mathrm{~h}$ se pasa sentado.

A continuación, se puso en marcha una intervención para promover la actividad física en la escuela Jean Jacques Rousseau (ubicada en el REP) durante el año escolar 2017/2018, para todos los niveles (desde el CP [6-7 años] hasta CM2 [10-11 años]). Se realizaron mediciones durante y después de esta intervención para comprobar su eficacia. La intervención también está en marcha en la Escuela Jules Verne durante el año escolar 2018/2019.

Los niveles de AF y de sedentarismo en los niños pueden depender de numerosos factores que, en el modelo socio-ecológico, se encuentran en diferentes esferas: personal (psicología), interpersonal (amigos, familia), organizativa (escuela), comunitaria (barrio, ciudad) y política (programas sanitarios implantados) (Bronfenbrenner, 1977).

Este año se pusieron en marcha varias iniciativas de intervención en relación con el modelo socio-ecológico:

1. Diseño del patio del recreo: trazado de las marcas del suelo.

2. Reorganización del servicio de comedor: el tiempo pasado en el comedor entre las $12 \mathrm{~h}$ y las $14 \mathrm{~h}$ se redujo para liberar tiempo de juego para los niños antes de las clases de la tarde.

3. Talleres de sensibilización para niños: se realizan en clase y en el recreo, para que tomen conciencia de la necesidad de un estilo de vida saludable.

4. Talleres de sensibilización para padres: también pretenden una toma de conciencia sobre la importancia de los comportamientos saludables y abordan los aspectos más prácticos de la actividad física así como el estilo de vida sedentario de los niños.

5. Formación para el profesorado: afecta a todos los niveles escolares. Se han programado un total de 4 reuniones de 2 horas, en las que se abordan diversas cuestiones. a) Tiempos de diálogo para reflexionar sobre los conceptos de comportamientos saludables. b) Consejos prácticos: aprendizaje de técnicas pedagógicas para que los niños sean más activos en el contexto escolar y reducir el tiempo sedentario, la inclusión del conocimiento relacionado con los comportamientos de salud en todas las materias académicas (interdisciplinariedad). c) Formación en técnicas de relajación (sofrología) para promover el bienestar de los niños y reducir el tiempo de sedentarismo. 
Los profesores también recibieron un folleto con diferentes ejercicios de relajación. También se les alentó a estar activos e incluso utilizaron sensores para conocer sus niveles de actividad física y sedentarismo.

Los profesores que experimentaron esta intervención en las escuelas participarán en la presentación para contar su experiencia. Compartirán sus conocimientos, ideas y vivencias.

Próximamente se realizarán análisis estadísticos para medir:

1. La eficacia de la intervención AF y TS en la escuela.

2. La relación entre actividad física, sedentarismo, capacidad de atención y éxito académico.

\section{Conclusión:}

Tras este estudio, se prevé mejorar los programas de intervención para ofrecerlos a otras escuelas en relación con los servicios educativos de la región, con el objetivo de aumentar la sensibilización de la mayoría del alumnado. Este estudio, cuyo objetivo es promover la actividad física en los niños, quiere generar conocimientos y habilidades en esta población para que adopten buenos hábitos en términos de salud que les acompañarán durante toda su vida.

\section{Bibliografía:}

Barnes, J., Behrens, T. K., Benden, M. E., Biddle, S., Bond, D., Brassard, P., ... \& Colley, R. (2012). Letter to the Editor: Standardized use of the terms "sedentary" and "sedentary behaviours". Applied Physiology Nutrition and Metabolism-Physiologie Appliquee Nutrition Et Metabolisme, 37(3), 540-542.

Bronfenbrenner, U. (1977). Toward an experimental ecology of human development. American psychologist, 32(7), 513-531.

Donnelly, J. E., Hillman, C. H., Castelli, D., Etnier, J. L., Lee, S., Tomporowski, P.,... \& Szabo-Reed, A. N. (2016). Physical activity, fitness, cognitive function, and academic achievement in children. Medicine \& Science in Sports \& Exercise, 48(6), 1197-1222. doi: 10.1249/mss.0000000000000901

Grélot, L. (2016). Activités physiques et sportives de l'enfant et de l'adolescent : des croyances aux recommandations sanitaires. Journal de Pédiatrie et de Puériculture, 29(2), 57-68. doi: 10.1016/j.jpp.2016.02.003 


\section{"Descansos" sedentarios Organización de la clase para disminuir el tiempo sedentario. Actividades "activas" en el aula}

Caroline Bernal ${ }^{1}$ y Nicolas Fabre ${ }^{1}$

${ }^{1}$ Université de Pau

\section{Resumen:}

La escuela tradicional clásica impone al alumnado estar sentados en clase para adquirir nuevos conocimientos y competencias. Este tiempo sentado en clase puede ser incluso de más de 5 horas, según los horarios de los centros.

Sin embargo, la acumulación de tiempo en una posición sedentaria podría tener efectos negativos en la salud, incluso desde la primera infancia. Por lo tanto, es primordial limitar, tanto como sea posible, el tiempo pasado en una actitud sedentaria y enseñar a los niños cómo limitar este tiempo sentado.

El niño aprende mediante experiencias vividas, y en particular a través de los movimientos realizados. Por lo tanto, está plenamente justificado utilizar métodos que hagan que los niños sean activos durante su aprendizaje y en sus vidas, lo que les lleva a experimentar.

¿Cómo reducir el tiempo de sedentarismo en clase y que los niños sean activos durante su aprendizaje?

Para reducir el tiempo de sedentarismo en clase se pueden emplear varias estrategias complementarias. Primero, veremos que el profesor puede hacer descansos o "breaks" en los que el niño estará activo durante diez minutos en clase, pero también veremos que la enseñanza puede adaptar la organización y los métodos de aprendizaje utilizados con la clase para disminuir el tiempo que el alumnado pasa sentado.

1. Hacer "breaks" de sedentarismo en clase en los momentos en los que el alumnado acumula demasiado tiempo sentado

\subsection{Hacer una pausa durante el aprendizaje en clase para realizar ejerci- cios de respiración, de relajación, de sofrología.}

Muchos adultos trabajan sentados en posición sedentaria. Les aconsejamos que se levanten cada hora y se estiren y caminen, para evitar los efectos negativos del sedentarismo. En la escuela, los niños están también sentados. El principio de pausas activas para los niños se basa en el mismo principio que para los adultos. Si el alumnado ha pasado ya mucho 
rato sentado, los profesores harán un descanso de 10 minutos durante la clase para realizar ejercicios de respiración, relajación, sofrología (llamadas "rutinas de prácticas corporales de bienestar"). Gracias a estos descansos, los niños no sólo se mueven y reducen el tiempo sedentario, sino que también se relajan antes de reanudar la clase: el aprendizaje relacionado con las clases de Lengua, Matemáticas, etc. se deja aparte durante el descanso activo.

Sin embargo, estos descansos activos deben incluir ejercicios físicos suaves o moderados para ser efectivos. De hecho, si la intensidad del esfuerzo es excesiva durante estas pausas, es contraproducente para el aprendizaje y para el éxito académico de los niños (Hillman \& Castelli, 2009). Demasiada intensidad conduce a la fatiga y reduce el rendimiento cognitivo.

Estas pausas activas se realizan en muchos países. El programa Energizers "Activity Promotion Laboratory" realizado en EEUU (Mahar et al., 2006), mostró que los niños que hicieron descansos activos en clase pasaron más tiempo concentrados y trabajando en su tarea, en comparación con los niños que no hicieron estos descansos.

\subsection{Realizar talleres de Lengua, Matemáticas, etc., para que los alumnos se muevan.}

Los descansos activos mencionados anteriormente dejan a un lado el aprendizaje relacionado con las asignaturas académicas, que se abordaban pensando en dejar un hueco para este momento de actividad. Varios estudios se han interesado por los efectos del ejercicio físico realizado al mismo tiempo que el aprendizaje. La clase de Matemáticas o Lengua continuará durante el descanso activo, pero mientras los alumnos se mueven. Algunas clases usan balones de fitness ("Swiss-balls" como apoyo para sentarse), o incluso cintas de correr. Sin embargo, los efectos de usar este material mientras se adquieren conocimientos y habilidades aún están en duda. Además, hay poca información sobre los efectos positivos en la lectura que pueda provocar en los alumnos de Primaria el uso de una bicicleta estática en clase. (Hillman \& Catelli, 2009).

Estos materiales (bicicletas, cintas de correr, etc.) imponen a los niños una práctica mecánica de la actividad física, sin aspecto lúdico. En este caso, es posible que el niño no sea capaz de medir la intensidad de su esfuerzo o que se disperse ante las múltiples tareas a realizar, lo que puede ir en detrimento de su aprendizaje. Además, este aspecto mecánico de la actividad física no le anima a estar activo fuera de este programa.

Se han establecido programas para hacer descansos activos durante las clases, pero incluyendo un enfoque lúdico a estos descansos. El programa TAKE 10 (Stewart et al., 2004) se realizó en EE.UU. en 50.000 aulas, lo que involucró a más de 1 millón de niños. El propósito de este programa fue incluir descansos activos en las asignaturas escolares, mientras se continuaba trabajando en clase mediante la realización de movimientos. 
Estos son algunos ejemplos concretos hechos en este programa.

- Los estudiantes realizan 10 saltos por cada respuesta correcta.

- Durante la clase de Matemáticas, los niños saltan cada 5 unidades (5-10-15-20), cada dos unidades (2-4-6-8) en orden creciente o decreciente.

- Para el aprendizaje de palabras y su ortografía, el alumnado puede tocar diferentes partes del cuerpo cuando se deletrean las palabras (GATO: G: tocarse la cabeza, A: tocarse los hombros, T: las rodillas, O: los pies).

- Al contar una historia o leer un libro, los alumnos pueden hacer movimientos en relación con la acción de la historia (paseo = vamos a pasear, aplaudir = vamos a aplaudir). También se pueden explicar conceptos complicados a los niños a través de los movimientos.

Este programa tiene un impacto positivo en el éxito académico de los niños de Primaria (Donnelly \& Lambourne, 2011), en el tiempo dedicado a trabajar en la tarea (Goh, Hanon, Webster, \& Hannon, 2016) así como en su comportamiento (Ribeiro \& Alves, 2014).

\section{Métodos de enseñanza que llevan al alumnado a moverse en clase.}

\subsection{Organización de talleres}

En la organización de la clase, existe la posibilidad de realizar talleres en diferentes mesas, durante los cuales los niños están de pie y pueden experimentar en cada taller. Cuando terminan un taller lo intercambian por otro y, por lo tanto, están en movimiento. Al final de los talleres, los alumnos recogen el material. Este principio se utiliza en la pedagogía Montessori. Montessori hace talleres en los que los niños experimentarán, aprenderán con sus propios gestos $\mathrm{y}$, por lo tanto, asociarán el movimiento con el pensamiento. Además, el espacio en el aula se administra para que el alumnado pueda moverse libremente entre los talleres. El niño trabajará su coordinación motora en el ambiente del taller creado por el adulto, lo que le permitirá alcanzar metas que tengan sentido para él. María Montessori considera que "el espíritu puede elevarse a través de la acción", atribuyendo un lugar fundamental al movimiento. Según ella, el niño tiene que desarrollar su cuerpo al mismo tiempo que su espíritu. Los niños usan sus músculos, manos y pies para explorar el mundo. Los niños no pueden crecer inmóviles y no es necesario estar en una posición especial para aprovechar la educación o para aprender. La coordinación de los músculos debe ser perfeccionada por el desarrollo cognitivo: estas ideas se unen a las actividades realizadas en las pausas activas donde se trabajan las diversas asignaturas académicas en movimiento. 


\subsection{Organización en la vida comunitaria.}

Para reducir el tiempo sedentario también puede ser interesante asignar tareas activas a los niños, permitiéndoles tomar responsabilidades. Por ejemplo, cada semana, un alumno puede hacerse cargo de la distribución de las fotocopias, otro puede tener la misión de hacer fotocopias durante esa misma semana, otro niño puede ir a ver cómo está el tiempo y la temperatura en el patio todas las mañanas, etc. Así, los niños tienen tareas activas para llevar a cabo. Esta organización de clase se usa especialmente en la pedagogía de Freinet. Los niños tienen tareas individualizadas pero también participan en el trabajo colectivo del grupo.

En conclusión, son posibles varios métodos para reducir el tiempo sedentario de los niños en clase. Todos estos métodos son complementarios $y$, por lo tanto, pueden permitir al profesorado variar su enfoque de trabajo con su alumnado.

\section{Bibliografía:}

Castelli, D. M., \& Hillman, C. H. (2012). Physical activity, cognition, and school performance: from neurons to neighborhoods. In Physical Activity Across the Lifespan (pp. 41-63). Springer New York.

Donnelly, J. E., \& Lambourne, K. (2011). Classroom-based physical activity, cognition, and academic achievement. Preventive medicine, 52, S36-S42.

Freinet, C. (1973). Les techniques Freinet de l'école moderne. Paris: A. Colin, coll. Bourrelier.

Goh, T. L., Hannon, J., Webster, C., Podlog, L., \& Newton, M. (2016). Effects of a TAKE 10! Classroom-Based Physical Activity Intervention on Third-to Fifth-Grade Children's On-task Behavior. Journal of Physical Activity and Health, 13(7), 712-718.

Mahar, M. T., Murphy, S. K., Rowe, D. A., Golden, J., Shields, A. T., \& Raedeke, T. D. (2006). Effects of a classroom-based program on physical activity and on-task behavior. Medicine and science in sports and exercise, 38(12), 2086.

Montessori, M., \& Bernard, G. (2016). La découverte de l'enfant: Pédagogie scientifique (Vol. 1). Desclée de Brouwer.

Ribeiro, R. Q., \& Alves, L. (2014). Comparison of two school-based programmes for health behaviour change: the Belo Horizonte Heart Study randomized trial. Public health nutrition, 17(6), 1195-1204. 


\section{Ejemplo de configuración del trabajo colaborativo.}

Pascal Legrain ${ }^{1}$ Y Guillaume Escalie ${ }^{1}$

'UF STAPS, Université de Bordeaux

\section{Resumen del taller:}

Este taller consiste en proporcionar a los asistentes un dispositivo de aprendizaje cooperativo Jigsaw (Aronson \& Patnoe, 1997). El principio pedagógico que subyace en este enfoque consiste en considerar que la experimentación de dispositivos cooperativos de aprendizaje para la formación por parte del profesorado puede facilitar su posterior implementación en el aula con el alumnado.

El dispositivo Jigsaw ("Grupo de expertos") tiene las siguientes características distintivas. Los participantes se organizan en equipos. El material o los recursos se dividen en bloques, y se invita a los miembros de cada equipo a distribuirse entre varios talleres para acceder eficazmente a todos los recursos. Como primer paso, cada miembro tiene la responsabilidad de aprender parte de la tarea en un grupo llamado "de expertos". En un segundo paso, se le pide que regrese a su equipo original para enseñar a sus compañeros lo que ha aprendido y recoger la información que generan sus compañeros.

El taller se basa en la actividad física Savate/Boxeo francés. Los participantes, agrupados en equipos de ocho, realizan por parejas un taller para aprender una técnica de pie (crochet o patada directa) o puño (chassé o fouetté). Al principio, los participantes son instruidos sobre la técnica que deben usar a través de un vídeo grabado en una tablet. En un segundo paso, los participantes, por parejas, practican esta técnica. En tercer lugar, el monitor realiza una actividad de observación-regulación animando a los participantes a preguntarse por las principales dificultades de aprendizaje encontradas en sus respectivos talleres. Finalmente, se les pide que se unan a su equipo para enseñar, como tutor, los conocimientos y las habilidades adquiridas, y para aprender, en calidad de alumno, los conocimientos y las habilidades aprendidas previamente por sus compañeros. El objetivo colectivo final del equipo es que cada miembro pueda producir una secuencia que integre las cuatro técnicas analizadas.

\section{Bibliografía:}

Aronson E. \& Patnoe S. (1997). The jigsaw classroom: Building cooperation in the classroom. New York: Addison-Wesley.

Escalié, G., Legrain, P., \& Lafont, L. (2018). L'apprentissage coopératif en " groupe d'experts » et la professionnalisation des futurs enseignants: un exemple en Education Physique et Sportive. Carrefours de l'éducation, 46, 161-176.

Legrain, P. (1998). Boxe française. Apprentissage et enseignement. Paris: Editions Revue EPS (coll. Les cahiers du sport). 


\title{
Teoría y práctica de la promoción de la AF: Encuentros Intercentros. "El Test de Responsabilidad”. Una estrategia previa a los Encuentros Intercentros
}

\author{
Paz Tierz', Gemma Bermejo², Raquel Pérez-Ordás ${ }^{2}$ y Eduardo Generelo ${ }^{2-3}$ \\ ${ }^{1}$ Instituto de Educación Secundaria Sierra de Guara. Huesca. \\ 2 CAPAS-Ciudad Huesca. \\ ${ }^{3}$ Facultad de Ciencias de la Salud y del Deporte. Universidad de Zaragoza.
}

\section{Resumen:}

El taller presenta y explica el "Test de Responsabilidad". Se trata de una situación de trabajo que utiliza cinco juegos cooperativos con objeto de estimular en el alumnado adolescente la capacidad de organizarse y cooperar para resolver problemas en grupo. El test supone un paso dentro del complejo proceso de empoderar a los sujetos para que sean los protagonistas de su propia práctica de actividad física. Los Encuentros Intercentros constituyen una situación de referencia dentro de este proceso.

\section{Introducción y contextualización:}

Deseamos que los grupos escolares sean capaces de participar con máxima autonomía en la vida de un centro. Organizar recreos, participar en comisiones, ser protagonistas de la resolución de conflictos, contribuir en la gestión de la biblioteca, organizar y participar en actividades deportivas y recreativas, etc. constituyen un aprendizaje básico para el objetivo general de formar ciudadanos participativos y críticos en la sociedad en la que se desenvolverán.

El "Test de Responsabilidad" es una herramienta para orientar acciones encaminadas a que un grupo escolar se implique y asuma responsabilidades en la actividad escolar.

El "Test de Responsabilidad" es una propuesta que se realiza como reto a un grupo escolar y que tienen que resolver en un tiempo determinado. El equipo de profesores y el propio alumnado observa el resultado de la prueba y se apoyarán en este y en el proceso desempeñado por el grupo para encuadrar desde el debate aquellas habilidades sociales y pautas de comportamiento grupal que les conducirá a enfrentarse con eficacia a problemas para los que sea fundamental la colaboración.

Participar en la organización de sus recreos, en Encuentros Intercentros o en viajes escolares serán, por ejemplo, situaciones de referencia para las cuales debe desplegarse todo un proceso en el que el test de responsabilidad es un punto de apoyo. 


\section{Descripción de la propuesta:}

El test consiste en proponer a un grupo escolar el reto de superar cinco pruebas colaborando entre toda la clase. Los objetivos reales que busca el profesorado son:

- Conocer el comportamiento de un grupo al enfrentarse a un problema colectivo.

- Identificar el papel que juegan en un grupo sus componentes.

- Hacer que el grupo tome conciencia también de los objetivos anteriores.

- Orientar diferentes habilidades sociales fundamentales para abordar empresas colectivas.

El proceso unido al test de responsabilidad conlleva una serie de acciones por parte del profesorado y el alumnado que se desglosan en:

1. Realización del test inicial

2. Trabajo cooperativo en clase sobre reflexión de lo sucedido en el desarrollo del test

3. Repetición del test

4. Concreción de los pasos a seguir para resolver los problemas planteados en la ejecución del test. Establecer códigos vinculados con habilidades sociales y grupales

5. Aplicación de las estrategias pactadas, con el objeto de empoderar al alumnado y motivar la autonomía para generar acciones propias (Encuentros Intercentros, recreos divertidos, salidas, etc.)

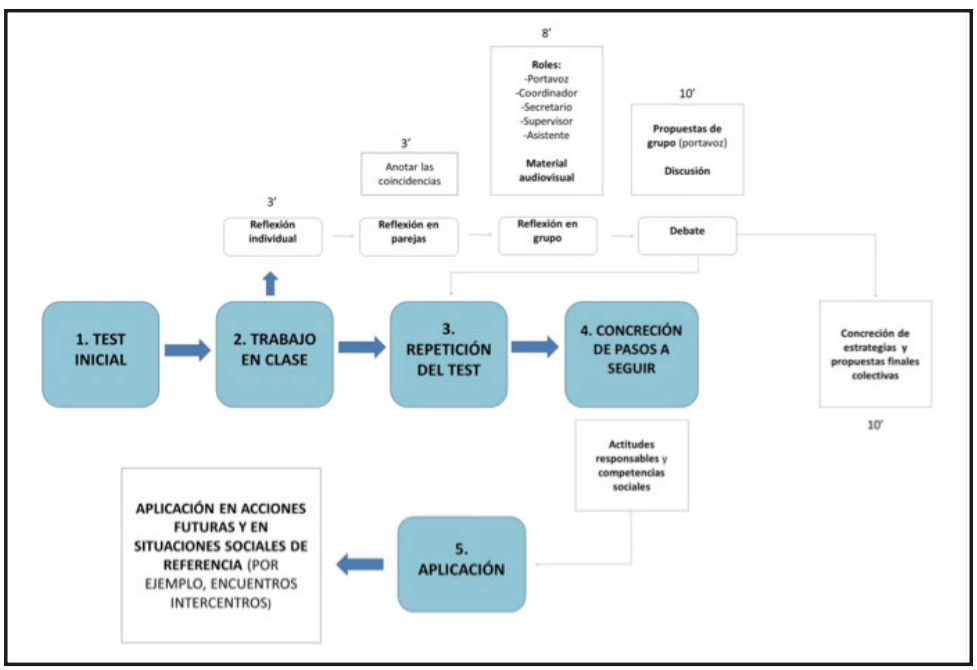




\section{Situaciones de aprendizaje claves:}

El proceso de trabajo consiste en lanzar el reto al grupo, comprobar si se consigue el objetivo del juego y discutir con el grupo las razones por las que se ha conseguido o no el éxito. El desarrollo del test se tiene que realizar en varias sesiones de trabajo y es fundamental que el seguimiento lo haga un mismo profesor o un equipo docente en el marco de una propuesta de intervención a ser posible de carácter transversal.

\section{Conclusión:}

En el trabajo para participar en un modelo como los Encuentros Intercentros el "Test de Responsabilidad" puede ser una estupenda herramienta para fomentar la cohesión del grupo y el empoderamiento.

\section{Bibliografía:}

Fraile, A. (2003). Actividad física y salud. Educación secundaria. Valladolid: Junta de Castilla y León.

Murillo, B., Sevil, J., Julián, J. A., \& Generelo, E. (2018). Sigue la Huella: Guide à l'usage des enseignants pour la promotions d'habitudes de vie saínes chez les élèves. CAPAS-Cité.

Santed, M. (2016). El valor del juego cooperativo en alumnos de $2^{\circ}$ de ESO. Trabajo Fin de Máster. Universidad de Zaragoza, Huesca. 


\title{
Teoría y práctica de la promoción de la AF: Encuentros Intercentros
}

\author{
José Antonio Julián ${ }^{1-2}$, Javier Sevil ${ }^{2}$, Alberto Aibar ${ }^{2-3}$ \\ ${ }^{1}$ Facultad de Ciencias Sociales y Humanas. Universidad de Zaragoza. \\ ${ }^{2}$ Grupo de Investigación EFYPAF. Universidad de Zaragoza. \\ ${ }^{3}$ Facultad de Ciencias Humanas y de la Educación. Universidad de Zaragoza.
}

\section{Resumen:}

Este taller aborda la puesta en práctica de la actividad físico-deportiva del voleibol como contenido de la Educación Física escolar a través de una propuesta de enseñanza-aprendizaje novedosa que se llama Smashball (http://es.smashbal.nl/). Esta propuesta está orientada a la participación de los estudiantes en una situación social de referencia escolar de la ciudad de Huesca, que recibe el nombre de "Encuentro Intercentros de voleibol".

\section{Introducción y contextualización:}

La ciudad de Huesca cuenta con una serie de encuentros físico-deportivos intercentros en los que participan la totalidad de los centros de Educación Secundaria de la ciudad de Huesca. Estos encuentros, que presentan un carácter lúdico festivo, pretenden convertirse en situaciones sociales de referencia para la construcción de los procesos de enseñanza aprendizaje de determinados contenidos de la Educación Física (EF) escolar. Los docentes aprovechan todo el potencial educativo de estos encuentros incluyéndolos en su programación y planteando las unidades didácticas correspondientes en las semanas previas a la realización de dichos encuentros. Uno de estos eventos al que acuden todos los alumnos de $2^{\circ}$ de ESO de la ciudad de Huesca es un torneo de voleibol 3x3. La reflexión metodológica del conjunto del profesorado sobre la enseñanza previa del voleibol, con objeto de poder favorecer una mayor participación de todo el alumnado, se vuelve fundamental dentro de una construcción social del deporte como actividad inclusiva e integradora.

\section{Descripción de la propuesta:}

El voleibol ha sido un contenido que tradicionalmente se ha enseñado desde un abordaje más tradicional donde la complejidad de determinados gestos técnicos dificulta en ocasiones su enseñanza a nivel escolar. Esta característica genera dificultades a la hora de plantear propuestas educativas donde la totalidad de los escolares puedan sentirse competentes y tener éxito en su práctica. El Smashball es una forma jugada de reciente 
creación por la Federación Holandesa de voleibol que pretende flexibilizar las reglas y el dinamismo de la actividad mediante la introducción del remate como elemento motriz clave del juego. La organización de su enseñanza en torno a este elemento del juego busca generar una práctica deportiva más inclusiva, participativa y accesible para todo el alumnado.

\section{Situaciones de aprendizaje claves:}

Este taller presenta una serie de situaciones de aprendizaje iniciales del Smashball más orientadas a la etapa de Educación Primaria. Concretamente se plantean tres situaciones de aprendizaje del Smashball (N1, N2, N3) que pretenden dar respuesta a aspectos principales de la enseñanza del voleibol.

1. Smashball N1: Situación inicial de $2 \times 2$ en la que se introduce el concepto de Smash (autolanzamiento y golpeo) como elemento fundamental de juego y alternativa a las dificultades técnicas que suele plantear el voleibol. En esta situación los jugadores pueden atrapar el balón con o sin bote y se puede avanzar con el balón agarrado.

2. Smashball N2: Situación 2x2 en la que se avanza en la construcción del juego incluyendo otras opciones de Smash (interacción con compañero) y a partir de la cual se genera la necesidad de trabajar la mejora técnica del gesto del remate para ser más efectivo en el juego. La interacción entre compañeros es mediante pases asistidos.

3. Smashball N3: Situación 2x2 en la que se avanza en la construcción del juego exigiendo la necesidad de incorporar un golpeo en el pase de colocación al compañero. Este golpeo genera la necesidad de incorporar tareas que fomenten el aprendizaje de determinados gestos técnicos como el pase de dedos y el saque de seguridad.

Se recomienda consultar el documento de Domingo \& Julián (2017), para obtener más información sobre la descripción de otras situaciones más propias de la etapa de Educación Secundaria y de aspectos generales de la secuencia didáctica de enseñanza del Smashball.

\section{Conclusión:}

El Smashball es una propuesta de enseñanza-aprendizaje del voleibol cuyo planteamiento encaja perfectamente en el diseño de encuentros intercentros donde se prime un ambiente lúdico y se favorezca el éxito de todos y cada uno de los participantes. Esta propuesta pretende favorecer una EF de calidad. 


\section{Bibliografía:}

Domingo, J., \& Julián, J. A. (2017). Secuencia didáctica para la introducción del voleibol-smashball en el contexto escolar: Una propuesta metodológica a los deportes de cancha dividida en contexto CRA. IV Jornadas de Educación Física +EF. Almudévar (Huesca). 25 de noviembre de 2017. 


\title{
Reflexiones sobre la organización de situaciones de enseñanza en EF con impacto sobre la salud: Trabajo intermitente de alta intensidad en deportes de equipo
}

\author{
Laurent Souriac ${ }^{1}$ \\ ${ }^{1}$ Département Staps Tarbes, Collège SSHS, \\ Université de Pau et des Pays de l’Adour
}

\section{Resumen:}

Desde hace mucho tiempo, los profesores de Educación Física reflexionan sobre el modo de organizar mejor las situaciones de enseñanza para incrementar los tiempos de esfuerzo de su alumnado. Trabajos recientes sobre los ejercicios intermitentes de alta intensidad (HIIE) han proporcionado unos puntos de vista distintos y complementarios que deben considerarse con interés. Este taller pretende, desde un contexto real de enseñanza de EF en baloncesto, reflexionar tanto sobre el aumento del tiempo de actividad física en una clase de EF como sobre un mejor planteamiento del tipo de actividades y sus impactos.

\section{Presentación:}

Los problemas relacionados con la salud superan con creces las prácticas de CP5 (competencia propia). Todas las APSA (actividades físicas, deportivas y artísticas) escolares desempeñan un papel esencial, especialmente los deportes colectivos (balonmano y baloncesto), porque siguen siendo una parte importante de las prácticas de enseñanza escolar. Pero conviene también preguntarse por la incorporación de los elementos didácticos más favorables a lo largo de la clase.

Durante el calentamiento y las situaciones de aprendizaje dirigidas a ciertas habilidades (pases, rebotes, regates, pasos, tiros, bloqueos...), el trabajo en forma de recorridos se usa con frecuencia y es interesante, porque reduce los tiempos de espera.

En situaciones con valencias tácticas, se potencia el trabajo colectivo en grupos pequeños y en aspectos particulares del juego (todos los tipos de 3c2, 4c3, combinaciones, apoyo defensivo...). En este caso, se trata de talleres específicos, más o menos diferenciados, que se ponen en marcha para optimizar el tiempo de práctica. 
Finalmente, en situaciones con valencias estratégicas y con todos los participantes, e incluyendo reversibilidad, la forma tradicional es el partido. Esos momentos son particularmente problemáticos, porque son complejos de gestionar y la mayoría de las veces son sinónimo de tiempos inactivos (muchos estudiantes y pocas pistas de juego).

En este taller de 12 participantes en un pequeño campo de $20 \times 12$, podremos comparar 3 situaciones estratégicas diferentes.

- 2 partidos de baloncesto 3 contra 3 de 3 minutos: 6 jugadores inactivos durante 3 minutos, frecuencia cardíaca (FC) por debajo del máximo.

- 1 serie de 6 minutos de ataques y defensas 3 contra 3 (el equipo ataca, luego defiende y luego sale para dejar su lugar al equipo que espera). En este caso, optimizamos el tiempo de práctica, todos los estudiantes juegan durante 6 minutos y están más involucrados desde un punto de vista informativo. Esta participación hace que sea posible organizar a los estudiantes en la tarea por un tiempo más largo, lo que permite reducir los tiempos de actividad perdidos. Desde un punto de vista energético, el trabajo ya no es continuo sino intermitente, lo que tiene la consecuencia de aumentar el tiempo pasado en una Fc más alta, porque la recuperación es incompleta.

- 1 serie de 6 minutos de ataques-defensas 3 contra 3 con alta intensidad. De acuerdo con la misma organización, se colocan conos a los lados del campo. Tan pronto como un equipo debe defender, los jugadores se retrasan porque tienen que rodear los conos por el exterior (fuera del campo). Este retraso de la defensa tiene el efecto de acelerar en gran medida la velocidad del ataque (ya que la zona a atacar está casi vacía). Aquí tenemos un juego de igualdad numérica basado en una serie de contraataques y repliegues defensivos rápidos. Este trabajo se asocia con la optimización del tiempo de actividad motora, una intensidad de la práctica que se aproxima o excede las capacidades aeróbicas máximas (trabajo en PMA). Muchos estudios nos muestran el impacto muy positivo de este tipo de trabajo.

- Aunque no se controle la velocidad de los jugadores, estamos muy cerca de un protocolo de trabajo en 15 " - 15 ", situándonos entre el $100 \%$ y el $120 \%$ de la VMA con valores cercanos a la Fc máxima. La siguiente tabla permite comprobar el perfil de los estudiantes que pueden beneficiarse de esta actividad. Si los alumnos tienen VMA más altas, el trabajo se puede llevar a cabo en un terreno grande de baloncesto (28m) (escuela secundaria, por ejemplo). En un contexto real de clase, es posible regular el equilibrio de fuerzas limitando o eliminando el retraso de algunos jugadores que tienen más dificultades. 


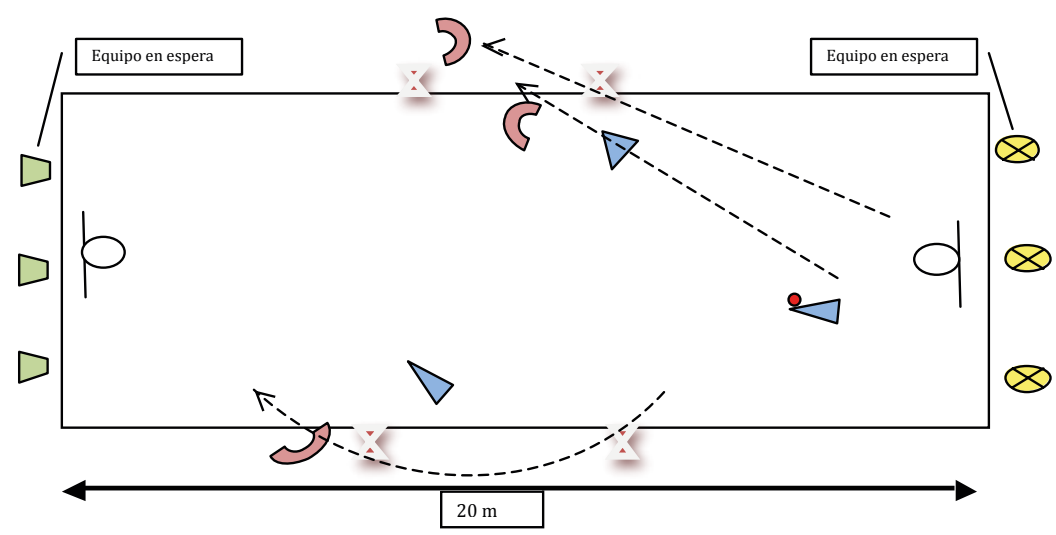

Este trabajo presentado en baloncesto $3 \mathrm{c} 3$ ha sido probado en baloncesto en 4c4, y en balonmano 4c4, 5c5 y 6c6. El impacto del trabajo se puede evaluar utilizando las escalas RPE (Borg, 1970) o ETL (Garçin et al., 1999)

\begin{tabular}{|c|c|c|c|c|c|c|c|c|c|}
\hline \multicolumn{10}{|c|}{ Exemple de duróes d'attaque-défense en BB pour une classe de cycle 4 en fonction de la VMA sur petit terrain } \\
\hline VMA en $\mathrm{km} / \mathrm{h} \rightarrow$ & 8 & 9 & 10 & 11 & 12 & 13 & 14 & 15 & 16 \\
\hline adaptations sco tps $40 \mathrm{~m}$ & 4,4 & 5,0 & 5,6 & 6,1 & 6,7 & 7,2 & 7,8 & 8,3 & 8,9 \\
\hline $100 \%$ VMA & 18,0 & 16,0 & 14,4 & 13,1 & 12,0 & 11,1 & 10,3 & 9,6 & 9,0 \\
\hline $110 \%$ VMA & 16,4 & 14,5 & 13,1 & 11,9 & 10,9 & 10,1 & 9,4 & 8,7 & 8,2 \\
\hline $120 \%$ VMA & 15,0 & 13,3 & 12,0 & 10,9 & 10,0 & 9,2 & 8,6 & 8,0 & 7,5 \\
\hline $130 \%$ VMA & 13,8 & 12,3 & 11,1 & 10,1 & 9,2 & 8,5 & 7,9 & 7,4 & 6,9 \\
\hline
\end{tabular}

\section{Bibliografía:}

Bond, B., Weston, K. L., Williams, C. A., \& Barker, A. R. (2017). Perspectives on high-intensity interval exercise for health promotion in children and adolescents. Open access journal of sports medicine, 8, 243-265.

Borg G. (1970). Perceived exertion as an indicator of somatic stress. Scand J Rehabil Med, 2(2), 92-98.

Garcin, M., Vandewalle, H., \& Monod, H. (1999). A new rating scale of perceived exertion based on subjective estimation of exhaustion time : A preliminary study. Int J Sports Med, 20(1), 40-43. 



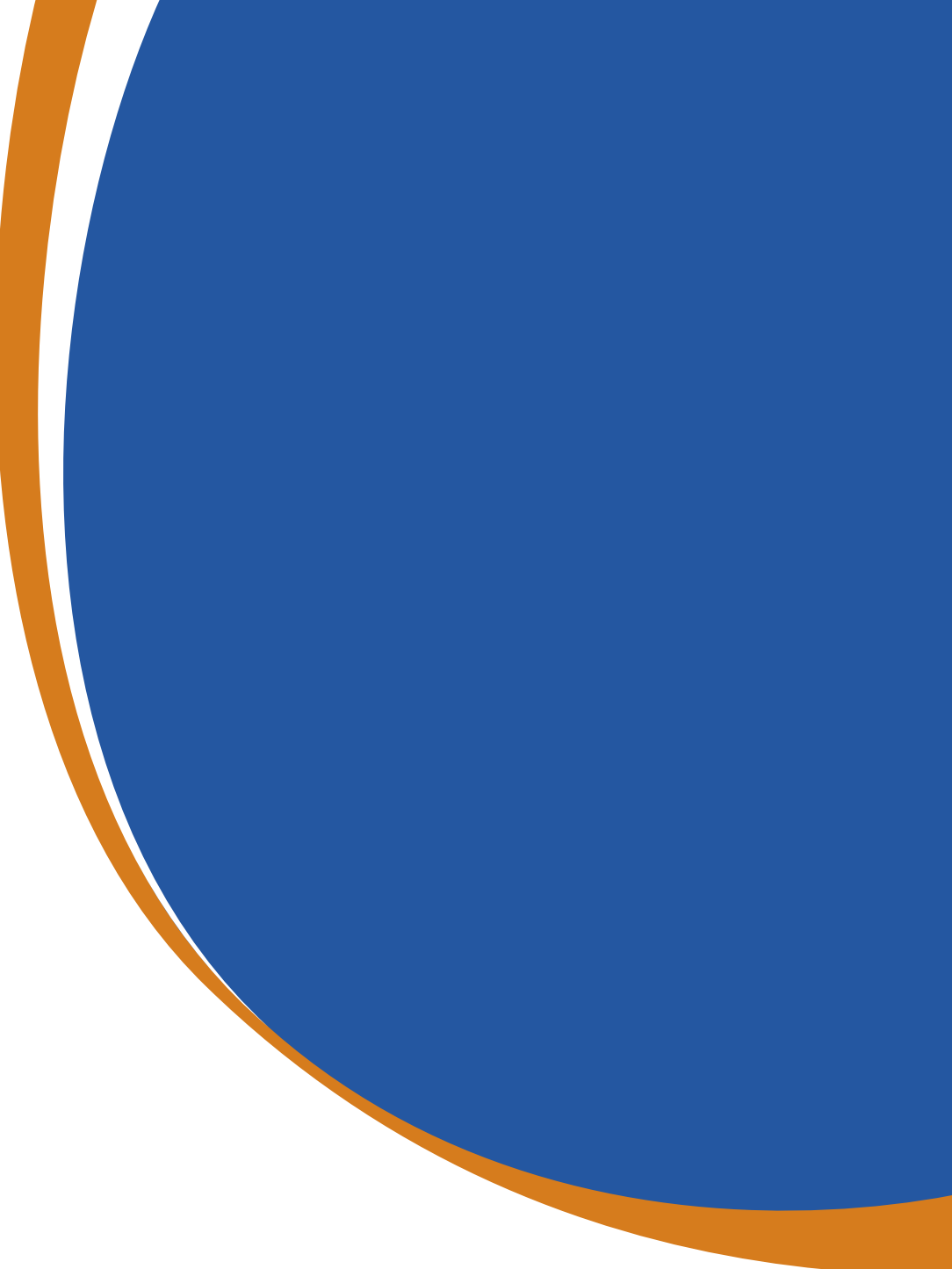

